\title{
Perioperative Assessment of Elderly Surgical Patients
}

\author{
Kenneth Ogan • Viraj A. Master • Daniel J. Canter
}

Published online: 26 February 2013

(C) Springer Science+Business Media New York 2013

\begin{abstract}
As the population continues to age, there will be a concomitant increase in the need for surgical intervention for a variety of disease processes for this group of patients. To date, surgical decision-making is overly subjective, and preoperative risk assessment tools are imprecise, lacking an ability to fully predict an elderly patient's physiologic reserve to withstand the intended insult of surgery. Frailty is an emerging concept that incorporates multiple functional, cognitive, and physiologic measurements to better grasp a patient's reserve. The preoperative measurement of frailty may offer an improved risk assessment tool to predict for adverse perioperative outcomes, especially in the elderly population.
\end{abstract}

Keywords Surgery $\cdot$ Morbidity $\cdot$ Mortality $\cdot$ Risk assessment $\cdot$ Frailty

\section{Introduction}

Much has changed since 500 B.C. when Euripides stated, "I hate the men who would prolong their lives by foods and drinks and charms of magic art. Perverting nature's course to keep off death...they ought, when they no longer serve the land to quit this life, and clear the way for youth." Due to the marked increase in the elderly population, today's physicians are now seeing and treating a growing proportion of older patients. From a surgical perspective, the surgeon must consider the delicate balance between the benefits of potentially

The information included in this manuscript has not been presented elsewhere.

K. Ogan · V. A. Master $\cdot$ D. J. Canter $(\bowtie)$

Department of Urology and Winship Cancer Institute,

Emory University School of Medicine,

1365 Clifton Rd. NE, Building B, Suite 1400,

Atlanta, GA 30322, USA

e-mail: daniel.j.canter@emory.edu life-saving/live-prolonging surgeries versus the risks of developing peri-operative complications that may negatively affect a patient's quality and quantity of life. This review will examine the inadequacies of current preoperative assessment tools and discuss novel ways to better assess an elderly patient's surgical risk.

\section{The Aging Population and the Physiology of Aging}

The number of people in the United States greater than 65 years of age is increasing, with the fastest increase seen in those 85 years old or greater. An aging population is also occurring worldwide; in some less developed countries, life expectancy actually increases by several hours every day. In the United States, life expectancy has risen from 47 years in 1900 to 77 years in 2001, and for the very aged, the life expectancy of a woman and man 85 years of age is 7 and 5.5 years, respectively $[1,2]$.

While beyond the scope of this review, we will highlight some of the common and predictable organ system changes that occur with aging that may impact peri-operative outcomes. Cardiac disease is the most common comorbid condition in the elderly [3]. Up to $80 \%$ of patients over 80 years of age have cardiovascular disease, accounting for over $40 \%$ of fatalities in those aged 65 years and older [4•]. Importantly, decreased cardiac reserve is sometimes difficult to identify because of compensatory factors that may mask cardiac dysfunction. Nonspecific findings such as generalized weakness, anorexia, fatigue, and insomnia may predominate in the elderly, especially with compensated heart failure. Therefore, it is important to inquire about functional capacity as individuals unable to climb a flight of stairs (4 METS), walk indoors around the house, or do light house work (1 MET) are at an increased risk for cardiac complications. From a pulmonary standpoint, the most important physiological changes 
associated with aging are a decrease in the static elastic recoil of the lung, a decrease in compliance of the chest wall, and a decrease in the strength of respiratory muscles [5]. These physiologic changes contribute to increased residual lung volumes (air trapping), increased functional residual capacity, and increased work of breathing. While studies have demonstrated that age itself is not an independent risk factor for needing mechanical ventilation after intensive care unit admission [6], there is an increased risk of an elderly patient being unable to wean off a ventilator due to these aforementioned age-related changes [7].

Renal function is also altered with age. These changes include a decrease in glomerular filtration rate (GFR), vascular dysautonomia, altered tubular handling of creatinine, reduction in sodium reabsorption and potassium secretion, and diminished renal reserve. Starting in the third decade of life, a person's GFR peaks and will then progressively decline at a rate of $8 \mathrm{ml} / \mathrm{min} / 1.73 \mathrm{~m}^{2}$ per decade. These alterations make older individuals more susceptible to renal insults, such as acute kidney injury, volume depletion and overload, and toxic reactions to drugs excreted by the kidneys [8]. Finally, the volume and weight of a person's brain decline with age at a rate of about $5 \%$ per decade after the age of 40 , with the slope becoming steeper after the age of 70 [9]. The resulting cognitive impairments may impact the abilities of elderly surgical patients to adequately care for themselves postoperatively.

As demonstrated by the above, aging results in a progressive loss in one's ability to respond to stressors, even intended, such as surgery, when compared to younger patients. These physiologic changes may result in an elderly patient's inability to recover from seemingly minor postoperative complications.

\section{Traditional Risk Assessment}

Many diseases predominantly affect the elderly, and more than half of all operations in the United States are performed on patients 65 years of age and older [10]. There have been numerous studies that have demonstrated that major surgical interventions in the elderly have similar outcomes compared to younger patients $[11,12]$. As such, surgical treatment decision-making in the elderly must weigh the potential benefits of surgery versus the potential morbidity and/or mortality of surgical intervention. Specifically, for elderly oncology patients, the United States National Comprehensive Cancer Network (NCCN) and the International Society of Geriatric Oncology (SIOG) have recommended that some form of geriatric assessment be conducted to help cancer specialists determine the best treatment for their older patients [13•, 14]. Regretfully, current preoperative risk assessment tools are not geared to specifically assess the elderly, and, even if they were, they are inadequate in reliably predicting postoperative outcomes in this functionally heterogenous group of patients. A comprehensive assessment for this population must be a multidimensional, multidisciplinary tool designed to evaluate an older person's functional ability, physical, cognitive, and mental health, and socio-environmental environment [15].

Traditional, available preoperative risk assessment tools to help guide treatment decision-making are the American Society of Anesthesiology Physical Status Classification System (ASA), Revised Cardiac Risk Index (RCRI), Eastern Cooperative Oncology Group (ECOG) performance status, and Physiological and Operative Severity Score for EnUmaration of Mortality and Morbidity (POSSUM). Although all of these tools attempt to standardize and codify the complexity or functional status of a given patient, they all lack prospective validation. The ASA classification system was originally developed in the 1940s and was only intended to be used as a statistical tool for retrospective analysis of hospital records. As such, the ASA scoring system does not measure operative risk; but rather, it globally assesses the degree of sickness or the physical state of a patient prior to anesthesia/surgery. However, a patient's ASA score is determined preoperatively based on subjective criteria that has been shown to have poor inter-observer reliability [16]. Nonetheless, many operating room personnel use this system as a means of quantifying surgical risk. For example, in a study of anesthesia personnel, a patient's ASA score was reported to be an indicator of anesthetic and surgical risk in $88 \%$ and $49 \%$, respectively [16]. Despite these findings, numerous studies have demonstrated that a patient's ASA score is not predictive of postoperative outcomes.

The RCRI was developed to determine cardiac/ischemic risk in noncardiac surgery. It was originally formulated from a single-center prospective cohort of 2,893 patients 50 years or older who were having major elective non-cardiac surgery. It consists of six equally weighted components: history of coronary artery disease, heart failure, or cerebrovascular disease, diabetes mellitus requiring insulin, renal insufficiency defined as a serum creatinine $>2 \mathrm{mg} / \mathrm{dL}$, and the need for high-risk non-cardiac surgery. In a systematic review by Ford and associates, they found that the RCRI had an area under the curve (AUC) of 0.75 at discriminating patients who developed complications, compared to those who did not after both vascular and nonvascular noncardiac surgery. The RCRI was less accurate in predicting perioperative mortality $(\mathrm{AUC}=0.62)$ [17]. However, since this index does not predict non-cardiac complications, most surgeons do not use this index as a risk assessment tool.

The ECOG Scale of Performance Status was originally developed in 1960 to create a common functional nomenclature for cancer patients in helping to determine which 
patients were physically suitable for entry into clinical trials. It is widely used to quantify the functional status of oncologic patients and carries important predictive ability in determining prognosis for a number of malignant conditions. A patient's ECOG performance status describes a patient's symptoms, as well as a patient's functional capacity, with respect to ambulatory status and ability for selfcare. As with the ASA scoring system, there is only moderate concordance (Kappa 0.44, $95 \%$ confidence limits 0.38-0.51) between observers, which worsens with a patient's increasing PS score (i.e, ECOG PS=3-4) [18].

POSSUM is a well-established scoring system for predicting postoperative morbidity and mortality in the general surgical population. Created by Copeland and colleagues between 1989 and 1991, the POSSUM scoring system consists of both a physiologic sub-score made up of 12 preoperative factors and an operative sub-score that includes six variables. When combined, a patient's POSSUM score can predict postoperative outcomes. The obvious drawback of this system is that part of the assessment occurs in the postoperative setting, and thus it is less useful in determining preoperative risk. In an effort to circumvent this problem, some investigators have demonstrated that the POSSUM physiologic sub-score alone is useful in predicting postoperative morbidity [19].

Due to the shortcomings of all of the above mentioned traditional risk assessment tools, as well as the fact that they are not intended specifically for the elderly, the Preoperative Assessment of Cancer in the Elderly (PACE) was developed, using elements of the Comprehensive Geriatric Assessment (CGA), to predict postoperative outcomes in the elderly. The CGA was created by geriatricians to assess the medical, psychological, functional, social, and environmental components of elderly persons, in order to develop a coordinated plan for treatment and long-term follow-up [20]. This instrument has been found to be predictive of morbidity and mortality for several chronic diseases and in a variety of clinical settings [20-22]. The validated instruments used with PACE include the following: Mini Mental Status Exam, Satariano's Modified Index of Comorbidities, Activities of Daily Living (ADL), Instrumental Activities of Daily Living (IADL), Geriatric Depression Scale, Brief Fatigue Inventory (BFI), ECOG performance status, ASA score, POSSUM, and Portsmouth POSSUM Modification. Pope and colleagues studied PACE to determine if it was predictive of postoperative outcomes in a cohort of 460 patients aged 70 years or older undergoing elective cancerrelated surgery $[23 \cdot \bullet]$. Within the components of PACE, IADL, BFI, and ASA were all significantly associated with comorbidities on multivariate analysis. Also, in a separate multivariate analysis, a moderate to severe $\mathrm{BFI}(\mathrm{RR}=1.46)$ and a dependent IADL $(\mathrm{RR}=1.36)$ were significantly associated with postoperative complications; and a dependent
ADL (RR 2.0) was associated with an increased length of stay [24]. While PACE does appear to have a robust potential as a preoperative risk assessment tool, the evaluation required is typically beyond the capacity of a busy surgical practice.

\section{Age and Peri-Operative Outcomes}

As the prior sections have detailed, available preoperative risk assessment tools to objectively predict a patient's surgical "fitness" are either lacking or too time-consuming. As such, surgical decision-making is overly subjective, rife with physician and patient biases that unduly guide treatment planning. Due to this lack of objective data, many important treatment decisions are made on incomplete data or instinct. Despite these flaws, the physician is faced with a population that is living longer, and once patients reach a certain age, their likelihood of still having a significant life expectancy is high. Thus, the decision to operate/treat must account for a patient's life span versus the natural history and severity of a given disease. Furthermore, the treatment decision-making process must somehow be able to adequately quantitate a patient's ability to survive and recover from a given surgery with an acceptable rate of morbidity and/or mortality. Finding or developing tools to fill this void are clearly paramount.

This clinical challenge is no more apparent than in the elderly cancer patient. According to the Cancer Incidence, Mortality and Prevalence in the European Union, $58 \%$ of cancers and $69 \%$ of cancer deaths occur in patients age 65 years or older [25]. Similarly, in the United States, according to a Surveillance Epidemiology and End Results (SEER) study, cancer incidence and mortality is $57 \%$ and $71 \%$, respectively, in patients older than 65 years of age [26].

Despite the increased incidence and prevalence of cancer in the elderly, there are numerous studies that demonstrate that this group of patients is being denied recommended screening [27], appropriate care [28], and enrollment in clinical trials [29] based on their chronologic age. This practice of "ageism", which the American Psychological Society in 2005 defined as a "prejudice toward, stereotyping of and/or discrimination against any person or persons directly and solely as a function of their having attained a chronological age that the social group defines as old" occurs despite the fact that numerous studies have shown that cancer-specific survival is independent of chronologic age for multiple visceral malignancies [30]. Thus, the dilemma exists that an aging population is more likely to harbor a malignancy, yet they are less likely to be diagnosed and treated appropriately, due to a bias based solely on chronologic age alone.

Yet, to date, one of the only pieces of objective data that reliably correlates with adverse peri-operative outcomes is increasing patient age. For example, in patients with invasive 
bladder cancer who require radical cystectomy and urinary diversion to effectively treat their cancer, population-based and institutional data sets show a clear increasing risk of 90day postoperative mortality with increasing age $[31 \bullet, 32 \bullet$, 33]. Furthermore, this relationship is also present in the elderly undergoing pancreatic and esophageal surgeries [34]. Unfortunately, what is lacking is the ability to definitively and objectively identify the vast majority of patients who will recover well from this surgery while sparing the remaining subset of patients from an adverse outcome.

It is important to note that this relationship between perioperative outcomes and age exists in other surgical populations that are not undergoing as extensive a surgical procedure as radical cystectomy, pancreatectomy, or esophagectomy, all of which include both an extirpative portion as well as a complex reconstructive component. Two important papers demonstrating this relationship are worthy of mention. Firstly, Polanczyk et al. examined the relationship among age, length of stay, and complications in patients undergoing noncardiac surgery. In this study of over 4,000 patients, the authors found a statistically significant relationship between increasing age and major or fatal peri-operative complications $(p<0.001)$ [35]. Patients older than 80 years were more likely to experience an in-hospital mortality than patients younger than 80 years $(\mathrm{OR}=3.5)$ [35]. Another finding of this study was that a patient's length of stay increased incrementally with increasing age. Similarly, Hamel et al. analyzed the Veterans Affairs National Surgical Quality Improvement Project to assess the relationship between outcomes and age. The findings of this analysis demonstrated a significantly higher rate of complications $(p<0.001)$ and mortality $(p<0.001)$ for patients older than 80 years as compared to patients younger than 80 years [36]. Specifically, for every year above the age of 80 years, a patient had a $5 \%$ increase in mortality [36]. Finally, this relationship between age and perioperative outcomes exists with even less major procedures, such as adrenalectomy. A recent analysis of the Nationwide Inpatient Sample assessing the morbidity and mortality among patients undergoing adrenalectomy revealed an overall $16.5 \%$ complication rate. Importantly, the authors found that there was a 41 and $60 \%$ increase in complication rates among patients $61-70$ years and $>70$ years old, when compared to patients younger than 60 years old [37].

\section{Surgical Frailty}

Although age in of itself is certainly a predictive factor for adverse surgical outcomes, it is not absolute. There are many elderly patients who undergo surgical interventions and recover without incident. Yet, there is no denying that within this group, there are patients who are potentially more at risk; thus, being able to better objectify and identify this subset of patients is imperative. Alternatively, simply deferring or denying care based on age alone would unnecessarily prevent a majority of patients from receiving appropriate and life-saving surgery.

Frailty is a relatively new concept that encompasses not only an elderly patient's chronologic age, but also a patient's ability to withstand physiologic stressors. Specifically, Fried et al. described frailty "as a biologic syndrome of decreased reserve and resistance to stressors, resulting from cumulative declines across multiple physiologic systems, and causing vulnerability to adverse outcomes" [38]. In this initial study, Fried and her colleagues operationalized the measurement of frailty across the following domains: shrinking (weight loss or sarcopenia), weakness, poor endurance/exhaustion, slowness, and low activity. Patients were considered frail if they tested positive for $\geq 3$ criteria, with patients positive for $1-2$ criteria considered intermediately frail. In this large cohort of patients, there was an increased incidence of the frail phenotype with increasing age: for example, only $3.2 \%$ of patients aged 65 70 years were deemed frail, whereas $16.3 \%$ and $25.7 \%$ of patients aged $80-84$ years and $85-89$ years, respectively, were deemed frail [38]. And, in those patients who met objective criteria for frailty, they had a statistically significant higher incidence of death, first hospitalization, first fall, worsening ADL disability, and worsening mobility disability at 3 and 7 years (all $p$ values $<0.0001$ ) [38].

Makary and colleagues established the importance of the relationship between frailty and surgical outcomes in postoperative patients 65 years or older. In this study of 594 patients, the authors reported complication rates of $11.4 \%$ and $43.5 \%$ in frail patients undergoing minor and major procedures, respectively. On multivariate analysis, frailty remained an independent predictor of surgical complications with intermediately frail and frail patients having a 2.06 and 2.54-times higher risk of complications compared to non-frail patients [39••]. Furthermore, frailty was an independent predictor of increasing length of stay and discharge to a skilled or assistedcare facility [39••]. The initial results found by Makary et al. have been further validated in other surgical populations. For example, in a small population $(n=57)$ of patients undergoing elective laparoscopic cholecystectomy, frailty was associated with an increased incidence of postoperative complications $(p=0.022)$, longer length of hospital stay $(p=0.023)$, and higher pain scales $(p=0.04)$ [40]. Similarly, in a study of patients $(n=83)$ aged 75 years or older undergoing surgery for colorectal cancer, frail patients were 4 and 3.5 times more likely to experience a major complication $(\mathrm{HR}=4.083,95 \%$ CI, $1.433-11.638)$ or a surgical/medical complication $(\mathrm{HR}=3.467,95 \% \mathrm{CI}, 1.113-10.795)[41 \bullet]$. Also, in another small study $(n=37)$ of women with a gynecologic malignancy undergoing resection, $27 \%$ and $16 \%$ of the patients were either intermediately frail or frail, respectively. The presence of any degree of frailty was a statistically significant predictor 
of a 30-day post-complication $(p=0.04)$ [42]. Finally, in a larger study of over 200 patients, the presence of frailty preoperatively predicted for postoperative discharge to an "institutional care facility", another important postoperative indicator [43].

These studies highlight that the traditional preoperative evaluation of elderly patients may be lacking. Although the sheer number of these studies is not large and the ones to date have relatively small numbers, our present methods of understanding a patient's ability to withstand an intended physiologic stress, such as surgery, may be unsophisticated. From the studies to date, it is evident that not all elderly surgical patients are created equal, and further refinement is needed. Even in younger patients, detecting frailty may be useful in identifying patients who require preoperative intervention/optimization or, perhaps, intensive postoperative care to help forestall adverse postoperative outcomes.

Due to this relative sparseness of measuring frailty in the present literature, we have just completed an initial study measuring frailty in a multidisciplinary surgical setting (urology, general surgery, and surgical oncology). Using Fried's criteria as a starting point, we supplemented our assessment with other measures, including preoperative biochemical serum values (e.g. albumin, serum creatinine, estimated GFR, etc.). We are now analyzing the relationship between our measures of preoperative frailty and postoperative outcomes. Our goal is to potentially create a more precise and potentially concise method to assess patients for preoperative frailty and aid in preoperative risk stratification. Although still early, our initial experience has shown us that measuring frailty is quick, inexpensive, able to be performed by clinic support staff, and easy to do with minimal disruption to the normal clinic flow.

\section{Conclusion}

There are an increasing number of elderly patients that are potential candidates for surgical procedures. Accurate preoperative assessment of the elderly surgical patient is paramount to identify those that will tolerate the insults of surgery. Current preoperative assessment tools are inadequate to discriminate between appropriate and inappropriate surgical candidates in this heterogeneous cohort. Failure to do so may either wrongly deny patients the standard of care treatment or put them at a greater, unnecessary risk for adverse peri-operative outcomes. As such, the relatively new concept of surgical frailty may better assess functional reserve in our older surgical population, and, thus, the ability of an elderly patient to tolerate the stress of surgery. Potentially, for those that are deemed too "frail" for surgery, interventions/alternatives to mitigate these peri-operative risks may be chosen.
Conflict of Interest Kenneth Ogan declares that he has no conflict of interest.

Viraj A. Master declares that he has no conflict of interest.

Daniel J. Canter declares that he has no conflict of interest.

\section{References}

Papers of particular interest, published recently have been highlighted as:

- Of Importance

-. Of major importance

1. Kearney N, Miller M, Paul J, Smith K. Oncology healthcare professionals' attitudes toward elderly people. Ann Oncol. 2000;11:599-601.

2. Arias E, Anderson RN, Kung HC, Murphy SL, Kochanek KD. Deaths: final data for 2001. Natl Vital Stat Rep. 2003;52:1-115.

3. Rosenthal RA, Kavic SM. Assessment and management of the geriatric patient. Crit Care Med. 2004;32:S92-S105.

4. - Colloca G, Santoro M, Gambassi G. Age-related physiologic changes and perioperative management of elderly patients. Surg Oncol. 2010;19:124-30. This article summarizes the pathophysiological changes that occur with aging. Understanding these changes allows the surgeon to better prevent and manage perioperative complications in the elderly.

5. Janssens JP, Pache JC, Nicod LP. Physiological changes in respiratory function associated with ageing. Eur Respir J. 1999;13:197-205.

6. Ely EW, Evans GW, Haponik EF. Mechanical ventilation in a cohort of elderly patients admitted to an intensive care unit. Ann Intern Med. 1999;131:96-104.

7. Thompson LF. Failure to wean: exploring the influence of age-related pulmonary changes. Crit Care Nurs Clin N Am. 1996;8:7-16.

8. Musso CG, Oreopoulos DG. Aging and physiological changes of the kidneys including changes in glomerular filtration rate. Nephron Physiol. 2011;119 Suppl 1:p1-5.

9. Mrak RE, Griffin ST, Graham DI. Aging-associated changes in human brain. J Neuropathol Exp Neurol. 1997;56:1269-75.

10. Robinson TN, Wu DS, Pointer LF, Dunn CL, Moss M. Preoperative cognitive dysfunction is related to adverse postoperative outcomes in the elderly. J Am Coll Surg. 2012;215:12-7. discussion 7-8.

11. Gardner GJ. Ovarian cancer cytoreductive surgery in the elderly. Curr Treat Options Oncol. 2009;10:171-9.

12. Berry MF, Hanna J, Tong BC, et al. Risk factors for morbidity after lobectomy for lung cancer in elderly patients. Ann Thorac Surg. 2009;88:1093-9.

13. • Extermann M, Aapro M, Bernabei R, et al. Use of comprehensive geriatric assessment in older cancer patients: recommendations from the task force on CGA of the International Society of Geriatric Oncology (SIOG). Crit Rev Oncol Hematol. 2005;55:241-52. This paper is a systematic review of the comprehensive geriaric assessment (CGA) in the evaluation of the elderly oncology patient. While the task force endorses the use of the CGA, there is no consensus on the specific tools or approach.

14. Balducci L, Yates J. General guidelines for the management of older patients with cancer. Oncology (Williston Park). 2000;14:221-7.

15. Elsawy B, Higgins KE. The geriatric assessment. Am Fam Physician. 2011;83:48-56.

16. Aronson WL, McAuliffe MS, Miller K. Variability in the American Society of Anesthesiologists Physical Status Classification Scale. AANA J. 2003;71:265-74. 
17. Ford MK, Beattie WS, Wijeysundera DN. Systematic review: prediction of perioperative cardiac complications and mortality by the revised cardiac risk index. Ann Intern Med. 2010;152:26-35.

18. Sorensen JB, Klee M, Palshof T, Hansen HH. Performance status assessment in cancer patients. An inter-observer variability study. Br J Cancer. 1993;67:773-5.

19. Egberts JH, Stroeh A, Alkatout I, et al. Preoperative risk evaluation of postoperative morbidity in IBD patients-impact of the POSSUM score. Int J Colorectal Dis. 2011;26:783-92.

20. Stuck AE, Siu AL, Wieland GD, Adams J, Rubenstein LZ. Comprehensive geriatric assessment: a meta-analysis of controlled trials. Lancet. 1993;342:1032-6.

21. Rich MW, Beckham V, Wittenberg C, Leven CL, Freedland KE, Carney RM. A multidisciplinary intervention to prevent the readmission of elderly patients with congestive heart failure. $\mathrm{N}$ Engl J Med. 1995;333:1190-5.

22. Extermann M, Hurria A. Comprehensive geriatric assessment for older patients with cancer. J Clin Oncol. 2007;25:1824-31.

23. •• Pope D, Ramesh H, Gennari R, et al. Pre-operative assessment of cancer in the elderly (PACE): a comprehensive assessment of underlying characteristics of elderly cancer patients prior to elective surgery. Surg Oncol. 2006;15:189-97. This study describes the preoperative assessment of the elderly (PACE), which incorporates a battery of validated assessment tools to determine the functional reserve of elderly cancer patients preoperatively. In this international prospective study, six of the seven components of PACE were associated with comorbidities.

24. Audisio RA, Pope D, Ramesh HS, et al. Shall we operate? Preoperative assessment in elderly cancer patients (PACE) can help. A SIOG surgical task force prospective study. Crit Rev Oncol Hematol. 2008;65:156-63.

25. Coebergh JW. Epidemiology in Europe. Eur J Cancer. 2001;37 Suppl 7:S217-27.

26. Ries LAG, Eisner MP, Kosary CL, et al., editors. SEER Cancer Statistics Review, 1975-2000: National Cancer Institute; 2003.

27. Peek ME. Screening mammography in the elderly: a review of the issues. J Am Med Wom Assoc. 2003;58:191-8.

28. Firat S, Bousamra M, Gore E, Byhardt RW. Comorbidity and KPS are independent prognostic factors in stage I non-small-cell lung cancer. Int J Radiat Oncol Biol Phys. 2002;52:1047-57.

29. Lang KJ, Lidder S. Under-representation of the elderly in cancer clinical trials. Br J Hosp Med (Lond). 2010;71:678-81.

30. Vercelli M, Capocaccia R, Quaglia A, Casella C, Puppo A, Coebergh JW. Relative survival in elderly European cancer patients: evidence for health care inequalities. The EUROCARE Working Group. Crit Rev Oncol Hematol. 2000;35:161-79.

31. - Morgan TM, Keegan KA, Barocas DA, et al. Predicting the probability of 90-day survival of elderly patients with bladder cancer treated with radical cystectomy. J Urol. 2011;186:829-34. This article demonstrates the predictive value of age in determining post-operative outcomes for patients undergoing radical cystectomy. In this cohort, approximately $20 \%$ of patients undergoing this major surgery died within 90 days of surgery.

32. • Liberman D, Lughezzani G, Sun M, et al. Perioperative mortality is significantly greater in septuagenarian and octogenarian patients treated with radical cystectomy for urothelial carcinoma of the bladder. Urology. 2011;77:660-6. This article re-emphasizes the impact of age on survival in radical cystectomy patients. It also highlights how difficult it can be to treat elderly patients with invasive bladder cancer.

33. Aghazadeh MA, Barocas DA, Salem S, et al. Determining factors for hospital discharge status after radical cystectomy in a large contemporary cohort. J Urol. 2011;185:85-9.

34. Finlayson E, Fan Z, Birkmeyer JD. Outcomes in octogenarians undergoing high-risk cancer operation: a national study. J Am Coll Surg. 2007;205:729-34.

35. Polanczyk CA, Marcantonio E, Goldman L, et al. Impact of age on perioperative complications and length of stay in patients undergoing noncardiac surgery. Ann Intern Med. 2001;134:637-43.

36. Hamel MB, Henderson WG, Khuri SF, Daley J. Surgical outcomes for patients aged 80 and older: morbidity and mortality from major noncardiac surgery. J Am Geriatr Soc. 2005;53:424-9.

37. Kazaure HS, Roman SA, Sosa JA. Adrenalectomy in older Americans has increased morbidity and mortality: an analysis of 6,416 patients. Ann Surg Oncol. 2011;18:2714-21.

38. Fried LP, Tangen CM, Walston J, et al. Frailty in older adults: evidence for a phenotype. J Gerontol A Biol Sci Med Sci. 2001;56: M146-56.

39. • Makary MA, Segev DL, Pronovost PJ, et al. Frailty as a predictor of surgical outcomes in older patients. J Am Coll Surg. 2010;210:901-8. This article is very important in that it shows how frailty can be applied to the surgical setting. In this study, the authors were able to show that frailty predicted adverse postoperative outcomes in older patients.

40. Lasithiotakis K, Petrakis J, Venianaki M, et al. Frailty predicts outcome of elective laparoscopic cholecystectomy in geriatric patients. Surg Endosc 2012. doi:10.1007/s00464-012-2565-0.

41. - Tan KY, Kawamura YJ, Tokomitsu A, Tang T. Assessment for frailty is useful for predicting morbidity in elderly patients undergoing colorectal cancer resection whose comorbidities are already optimized. Am J Surg. 2012;204:139-43. This article further underlines the impact of preoperative frailty on postoperative outcomes. In this report, the authors examine the role of frailty in a surgical subset of patients.

42. Courtney-Brooks M, Tellawi AR, Scalici J, et al. Frailty: an outcome predictor for elderly gynecologic oncology patients. Gynecol Oncol. 2012;126:20-4.

43. Robinson TN, Wallace JI, Wu DS, et al. Accumulated frailty characteristics predict postoperative discharge institutionalization in the geriatric patient. J Am Coll Surg. 2011;213:37-42. discussion 42-44. 